Sección Agrícola / Agriculture

Artículo de investigación / Research paper

\title{
Números cromosómicos en insectos de Argentina. I. Caracterización citogenética en 15 especies de importancia económica
}

\author{
Chromosome numbers in insects of Argentina. I. Cytogenetic characterization of 15 \\ species with economic importance
}

\author{
VALERIA DE LOS ÁNGELES PÁEZ; ALDO R. ANDRADA²; MARÍA M. MORENO-RUIZ- \\ HOLGADO $^{3}$; GABRIELA M. SILENZI-USANDIVARAS ${ }^{4}$; ANDREA OVIEDO ${ }^{5}$; \\ GRACIELA E. RUIZ-DE-BIGLIARDO ${ }^{6}$
}

\begin{abstract}
${ }^{1}$ Licenciada, Instituto de Genética, Fundación Miguel Lillo, Miguel Lillo 251 (4000), San Miguel de Tucumán, Tucumán, Argentina, paezvaleria@hotmail. com, https://orcid.org/0000-0002-3213-3890. ${ }^{2}$ Licenciado, Instituto de Genética, Fundación Miguel Lillo, Miguel Lillo 251 (4000), San Miguel de Tucumán, Tucumán, Argentina, arandrada@lillo.org.ar, https://orcid.org/0000-0002-3520-1406. ${ }^{3}$ Licenciada, Facultad de Ciencias Naturales e I. M. L., Unidad Ejecutora Lillo (UEL-CONICET), Miguel Lillo 205 (4000), San Miguel de Tucumán, Tucumán, Argentina, macarena_mrh@hotmail.com, https://orcid.org/0000-00015512-0438. ${ }^{4}$ Licenciada, Instituto de Genética, Fundación Miguel Lillo, Miguel Lillo 251 (4000), San Miguel de Tucumán, Tucumán, Argentina, gmsilenzi@, lillo.org.ar, https://orcid.org/0000-0002-4519-8503. ${ }^{5}$ Magister, Facultad de Ciencias Naturales e I. M. L., Unidad Ejecutora Lillo (UEL-CONICET), Miguel Lillo 205 (4000), San Miguel de Tucumán, Tucumán, Argentina, andreavfoviedo@gmail.com, https://orcid.org/0000-0002-4262-7798. ${ }^{6}$ Magister, Instituto de Genética, Fundación Miguel Lillo y Facultad de Ciencias Naturales e I. M. L., Unidad Ejecutora Lillo (UEL-CONICET), Miguel Lillo 205,251 (4000), San Miguel de Tucumán, Tucumán, Argentina, grabigliardo@hotmail.com, https://orcid.org/0000-0001-9346-7010.
\end{abstract}

Autor para correspondencia: Valeria de los Ángeles Páez, Licenciada, Instituto de Genética, Fundación Miguel Lillo, Miguel Lillo 251 (4000), San Miguel de Tucumán, Tucumán, Argentina, paezvaleria@hotmail.com, https:// orcid.org/0000-0002-3213-3890.

Citación sugerida / Suggested citation: PÁEZ, V. D. L. A.; ANDRADA, A. R.; MORENO RUIZ HOLGADO, M. M.; SILENZI USANDIVARAS, G. M.; OVIEDO, A.; RUIZ DE BIGLIARDO, G. E. 2020. Números cromosómicos en insectos de Argentina. I. Caracterización citogenética en 15 especies de importancia económica. Revista Colombiana de Entomología 46 (1): e8536. https://doi. org/10.25100/socolen.v46i1.8536

Recibido: 1-mar-2018

Aceptado: 14 -jun-2019

Publicado: 9-jul-2020

Revista Colombiana de Entomología ISSN (Impreso): 0120-0488

ISSN (En línea): 2665-4385

http://revistacolombianaentomologia.univalle.edu.co/

Open access

Publicadores / Publishers:

Sociedad Colombiana de Entomología

SOCOLEN (Bogotá, D. C., Colombia)

http://www.socolen.org.co

Universidad del Valle (Cali, Colombia)

http://www.univalle.edu.co/

(C) 2020 Sociedad Colombiana de Entomología - SOCOLEN y Universidad del Valle - Univalle
Resumen: En el presente trabajo se informan los números cromosómicos somáticos y/o gaméticos de 15 especies de insectos presentes en Argentina, que se caracterizan por su importancia económica como plagas de cultivos o controladores de plagas agrícolas, pertenecientes a los siguientes órdenes y familias: Neuroptera, Chrysopidae: Ceraeochrysa cincta $(\mathrm{n}=5+\mathrm{XY})$, Ceraeochrysa paraguaria $(\mathrm{n}=5+\mathrm{XY})$, Chrysoperla argentina $(\mathrm{n}=5$ $+\mathrm{XY})$, Chrysoperla asoralis $(\mathrm{n}=5+\mathrm{XY})$, Chrysoperla externa $(\mathrm{n}=5+\mathrm{XY})$, Leucochrysa cruentata $(\mathrm{n}=7+\mathrm{XY})$ y Plesiochrysa elongata $(\mathrm{n}=5+\mathrm{XY})$, Coleoptera, Coccinellidae: Cycloneda sanguinea $\left(\mathrm{n}=9+\mathrm{XY}_{\mathrm{p}}\right)$, Eriopis connexa $\left(\mathrm{n}=9+\mathrm{XY}_{\mathrm{p}}\right)$, Harmonia axyridis $(\mathrm{n}$ $=7+\mathrm{XY})$, Hippodamia convergens $(\mathrm{n}=9+\mathrm{XY})$ y Melyridae: Astylus atromaculatus $(\mathrm{n}=$ $\left.8+\mathrm{XY}_{\mathrm{p}}\right)$, Dermaptera, Forficulidae: Doru lineare $(2 \mathrm{n}=20)$, D. luteipes $(2 \mathrm{n}=20)$ y Diptera, Ulidiidae: Euxesta eluta $(\mathrm{n}=5+\mathrm{XY})$. Por primera vez, se cita información cromosómica para seis especies, incluyendo un número cromosómico gamético adicional para Euxesta eluta. Además, se da a conocer en Astylus atromaculatus un número cromosómico diferente a los recuentos previamente reportados.

Palabras clave: Chrysopidae, Coccinellidae, Forficulidae, Melyridae, números cromosómicos, sistemas cromosómicos de determinación del sexo.

Abstract: In the present study, the somatic and gametic chromosome numbers in fifteen species of insects from Argentina are reported. Said species are characterized for their economic importance as pest of crops or as agricultural pest controllers. The species belonged to the following orders and families: Neuroptera, Chrysopidae: Ceraeochrysa cincta $(\mathrm{n}=$ $5+\mathrm{XY})$, Ceraeochrysa paraguaria $(\mathrm{n}=5+\mathrm{XY})$, Chrysoperla argentina $(\mathrm{n}=5+\mathrm{XY})$, Chrysoperla asoralis $(\mathrm{n}=5+\mathrm{XY})$, Chrysoperla externa $(\mathrm{n}=5+\mathrm{XY})$, Leucochrysa cruentata $(\mathrm{n}=7+\mathrm{XY})$ y Plesiochrysa elongate $(\mathrm{n}=5+\mathrm{XY})$, Coleoptera, Coccinellidae: Cyclonela sanguinea $(\mathrm{n}=9+\mathrm{XY})$, Eriopis connexa $(\mathrm{n}=9+\mathrm{XY})$, Harmonia axyridis $(\mathrm{n}=7+\mathrm{XY})$, Hippodamia convergens $(\mathrm{n}=9+\mathrm{XY})$, y Melyridae: Astylus atromaculatus $\left(\mathrm{n}=8+\mathrm{XY}_{\mathrm{p}}^{\mathrm{p}}\right)$, Dermaptera, Forficulidae: Doru lineare $(2 n=20)$ and D. luteipes $(2 n=20)$, Diptera, Ulidiidae: Euxesta eluta $(\mathrm{n}=5+\mathrm{XY})$. Chromosomal information for six species and additional gametic chromosome number for Euxesta eluta are established for the first time. In addition, a new chromosome number is cited for Astylus atromaculatus that is different to the one previously reported.

Keywords: Chrysopidae, Coccinellidae, Forficulidae, Melyridae, chromosomes numbers, chromosomal systems of sex determination.

\section{Introducción}

La clase Insecta contiene una gran diversidad específica, con más de 1.000 .000 de especies descritas distribuidas en 32 órdenes (Virgilio et al. 2010; Pacheco et al. 2014). Los sistemas genéticos de determinación del sexo en insectos son 
heterogéneos y es posible distinguir tres tipos básicos: con machos diploides (diplodiploidía), con machos haploides (haplodiploidía) y aquel que puede ocurrir sin machos o telitoquía (Normark 2003). Asimismo, los insectos presentan un amplio rango de números cromosómicos. Ejemplo de ello, pueden ser los himenópteros, para los cuales se citan números cromosómicos muy bajos, $2 \mathrm{n}=2 \mathrm{y} \mathrm{n}=1(q / \hat{\sigma}$, respectivamente) en Myrmecia pilosula F. Smith, 1858 y $M$. croslandi Taylor, $1991 \mathrm{y}$, asimismo, tan altos como $2 \mathrm{n}=120$ en Dinoponera lucida Emery, 1901 (Crosland y Crozier 1986; Mariano et al. 2011).

En el presente trabajo, se realizaron estudios citogenéticos a 15 especies de importancia económica pertenecientes a los órdenes Coleoptera, Dermaptera, Diptera y Neuroptera,

Tabla 1. Orden, familias y especies estudiadas. Números cromosómicos somáticos (2n) y gaméticos (n), sistema sexual, sitios de muestreo y antecedentes bibliográficos. El signo (*) representa primeros registros para el género, el signo $(+)$ representa los primeros recuentos para las especies

\begin{tabular}{|c|c|c|c|c|c|}
\hline Taxón & 2n & $\mathbf{n}$ & $\begin{array}{l}\text { Cromosomas } \\
\text { sexuales }\end{array}$ & Sitios de muestreo/Localidades & $\begin{array}{l}\text { Antecedentes } \\
\text { bibliográficos }\end{array}$ \\
\hline \multicolumn{6}{|l|}{$\begin{array}{l}\text { Orden Neuroptera } \\
\text { Familia Chrysopidae }\end{array}$} \\
\hline Ceraeochrysa cincta (Schneider, 1851) & 12 & 6 & $\begin{array}{l}\mathrm{XY} \\
\mathrm{XY}\end{array}$ & $\begin{array}{l}\text { ARGENTINA: Prov. Corrientes, Dpto. Bella Vista, 30-VIII- } \\
\text { 2012, } 28^{\circ} 26^{\prime} 51^{\prime \prime S}, 58^{\circ} 58^{\prime} 59^{\prime \prime O} \text {. } \\
\text { BRASIL: Prov. San Pablo, Dpto. Jaboticabal }\end{array}$ & Lopes (2012) \\
\hline Ceraeochrysa paraguaria (Navás, 1920) & & 6 & $\mathrm{XY}$ & 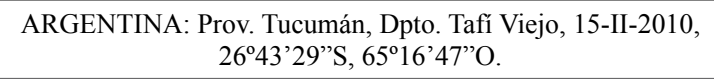 & \\
\hline \multirow{2}{*}{$\begin{array}{l}\text { Chrysoperla argentina (González Olazo } \\
\text { \& Reguilón, 2002) }\end{array}$} & & 6 & $\mathrm{XY}$ & $\begin{array}{l}\text { ARGENTINA: Prov. Tucumán, Dpto. Capital, Jardín Botánico } \\
\text { de la F.M.L. }\end{array}$ & \multirow{2}{*}{$\begin{array}{l}\text { Andrada et al. } \\
\qquad(2012)\end{array}$} \\
\hline & & 6 & $\mathrm{XY}$ & ARGENTINA: Prov. Tucumán, Dpto. Leales, 15-IV-2006. & \\
\hline Chrysoperla asoralis (Banks, 1915) + & & 6 & $\mathrm{XY}$ & $\begin{array}{l}\text { ARGENTINA: Prov. La Rioja, Dpto. Capital, 2-IV-2011, } \\
29^{\circ} 25^{\prime} 42^{\prime \prime} \mathrm{S}, 66^{\circ} 52^{\prime} 05^{\prime \prime} \mathrm{O} .\end{array}$ & \\
\hline Chrysoperla externa (Hagen, 1861) & & $\begin{array}{l}6 \\
6\end{array}$ & $\begin{array}{l}\mathrm{XY} \\
\mathrm{XY}\end{array}$ & $\begin{array}{l}\text { ARGENTINA: Prov. Tucumán, Dpto. Burruyacú, 6-III-2009. } \\
\text { ARGENTINA: Prov. Tucumán, Dpto. Burruyacú, 8-III-2007 }\end{array}$ & $\begin{array}{l}\text { Andrada et al. } \\
\text { (2012) }\end{array}$ \\
\hline Leucochrysa cruentata (Schneider, 1851) & 16 & 8 & $\mathrm{XY}$ & 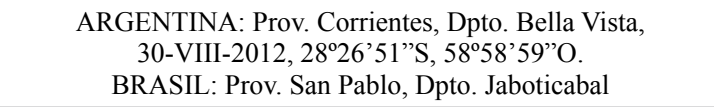 & Lopes (2012) \\
\hline Plesiochrysa elongata (Navás, 1913) & & 6 & $\mathrm{XX}: \mathrm{XY}$ & ARGENTINA: Prov. Tucumán, Dpto. Capital, 5-III-2010. & \\
\hline
\end{tabular}

\section{Orden Coleoptera}

Familia Coccinellidae

Cycloneda sanguinea (Linnaeus, 1763)

$10 \quad \mathrm{Xy}_{\mathrm{p}} \quad$ ARGENTINA: Prov. Tucumán, Dpto. Lules, X-2011,

\begin{tabular}{lll} 
& 10 & $\mathrm{XY}$ \\
20 & 10 & $\mathrm{Xy}$ \\
\hline & 10 & $\mathrm{Xy}$
\end{tabular}

Eriopis connexa (Germar, 1824)

\begin{tabular}{ccc}
20 & 10 & $\mathrm{Xy}_{\mathrm{p}}$ \\
\hline & 8 & $\mathrm{Xy}_{\mathrm{p}}$
\end{tabular}

Harmonia axyridis (Pallas, 1772)

$\begin{array}{lll}16 & & \mathrm{Xy} \\ 16 & & \mathrm{XX} \\ & & \mathrm{Xy}_{\mathrm{p}} \\ & 10 & \end{array}$
$26^{\circ} 51^{\prime} 01$ 's, 6517'02”O

Maffei et al. BRASIL: Prov. Minas Gerais, Dpto. Amoras (2004)

ARGENTINA: Prov. Tucumán, Dpto. Lules, X-2011, Maffei et al. 26⒌ 51 '01'S, 65'17'02'O BRASIL: Prov. Minas Gerais, Dpto. Amoras

ARGENTINA: Prov. Tucumán, Dpto. Lules, IV-2012, 26 51'01's, 65¹7'02"O. et al. (2001)

Hippodamia convergens (GuerinMeneville, 1842) *

JAPÓN: Prov. Honshu, Dpto. Machida-shi.

\section{Familia Melyridae}

Astylus atromaculatus (Blanchard, 1843)

$9 \quad \mathrm{Xy}_{\mathrm{p}}$

ARGENTINA: Prov. Tucumán, Dpto. Lules, IV-2012, $26^{\circ} 51$ '01'S, 65'17'02”O

\section{Orden Dermaptera}

Familia Forficulidae

\begin{tabular}{|c|c|c|c|c|}
\hline Doru lineare (Eschs, 1822) & 20 & XY & $\begin{array}{l}\text { ARGENTINA: Prov. Tucumán, Dpto. Trancas. } 26^{\circ} 32^{\prime} 02^{\prime} \mathrm{S} \text {, } \\
65^{\circ} 17^{\prime} 25^{\prime} \mathrm{O} \text {. } \\
\text { ARGENTINA: Prov. Tucumán, Dpto. Tafí Viejo, El Cadillal, } \\
26^{\circ} 40^{\prime} 48^{\prime \prime} \mathrm{S}, 65^{\circ} 16^{\prime} 00^{\prime} \mathrm{O} \text {. } \\
\text { Dpto. Lules, El Manantial } 26^{\circ} 49^{\prime} 50^{\prime \prime S}, 65^{\circ} 16^{\prime} 59^{\prime} \mathrm{O}\end{array}$ & $\begin{array}{l}\text { Andrada et al. } \\
\text { (2016) }\end{array}$ \\
\hline D. luteipes (Scudder, 1876) + & 20 & $X Y$ & $\begin{array}{l}\text { ARGENTINA: Prov. Tucumán, Dpto. Yerba Buena. } \\
\text { 6-VII-2017. }\end{array}$ & \\
\hline
\end{tabular}

\section{Orden Diptera}

Familia Ulidiidae

Euxesta eluta (Loew, 1868)

$\begin{array}{lll} & 6 & \mathrm{XY} \\ 12 & & \mathrm{XY}\end{array}$



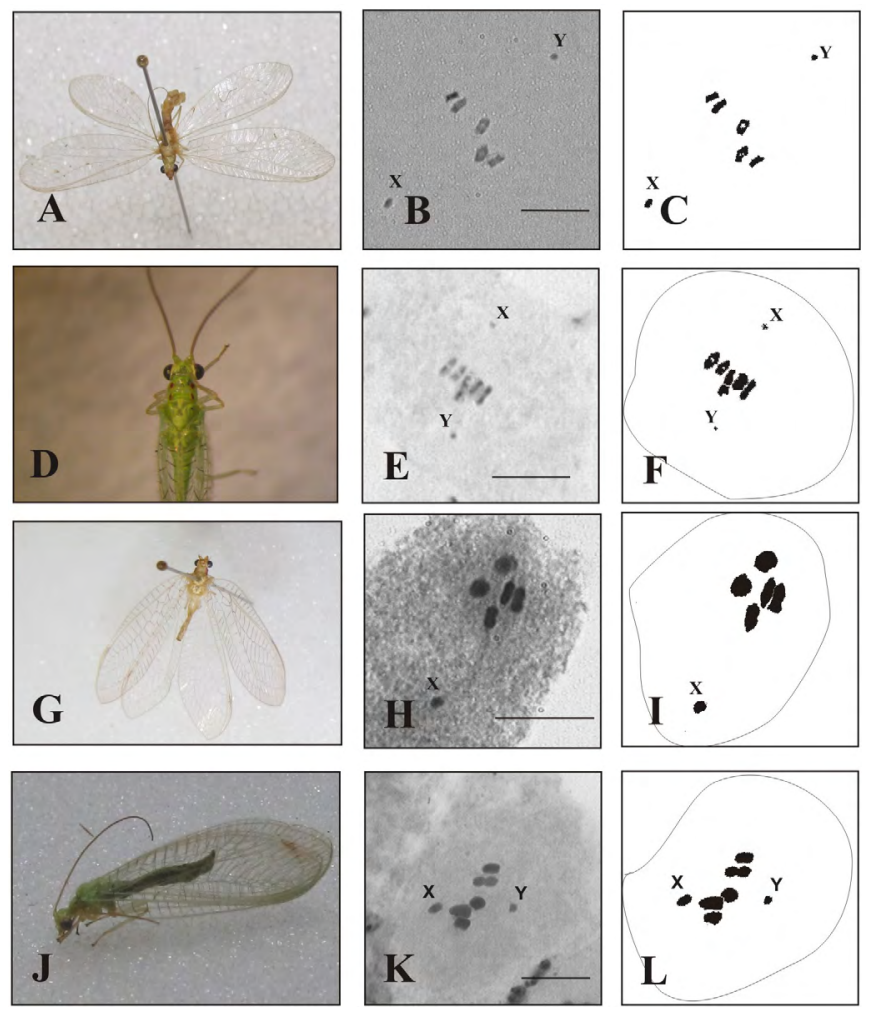

Figura 1. Neuroptera. A-C Ceraeochrysa cincta: A. Aspecto general de un individuo de colección. B. Metafase I $n=5+X Y$. C. Representación gráfica de Fig. 1C. D-F Ceraeochrysa paraguaria: D. Aspecto general del tórax. E. Metafase I n $=5+$ XY. F. Representación gráfica Fig. 1E. G-I Chrysoperla argentina: G. Aspecto general de un individuo de colección. H. Metafase I n $=5+$ XY. I. Representación gráfica Fig. 1H. J-L C. asoralis: J. Aspecto general de un individuo de colección. K. Metafase I n $=5+$ XY. L. Representación gráfica. Escala $=10 \mu \mathrm{m}$.

los cuales pueden actuar en los cultivos agrícolas como insectos beneficiosos o perjudiciales, en el primer caso como controladores biológicos de plagas en su rol de depredadores y en la última instancia como insectos fitófagos (Brindle y Quintero Arias 1992; Mariani 1996, 1998; Romero Sueldo y Virla 2005, 2009; Romero Sueldo et al. 2005, 2010; Andrada et al. 2012).

Tomando en cuenta el gran número de especies de insectos en la naturaleza, la variación existente en sus sistemas genéticos de determinación del sexo y el hecho de que los recuentos cromosómicos conocidos hasta el momento son muy escasos, se planteó como objetivo analizar citogenéticamente las especies que habitan Argentina, principalmente el noroeste argentino (NOA) dando a conocer el número gamético y/o somático y sistema sexual en 15 especies. El presente reporte es el primero de una serie periódica, destinada a estudiar las características cromosómicas de los insectos de Argentina con escasa o carente de información citogenética, tanto para especies con importancia económica como para las que actualmente no son consideradas económicamente valorables.

\section{Materiales y métodos}

El material de estudio se recolectó con red entomológica (salvo Euxesta eluta (Loew, 1868)) en poblaciones naturales y/o en zonas de cultivos (campo de maíz y cítricos), de las provincias de Corrientes y Tucumán, Argentina (Tabla 1).
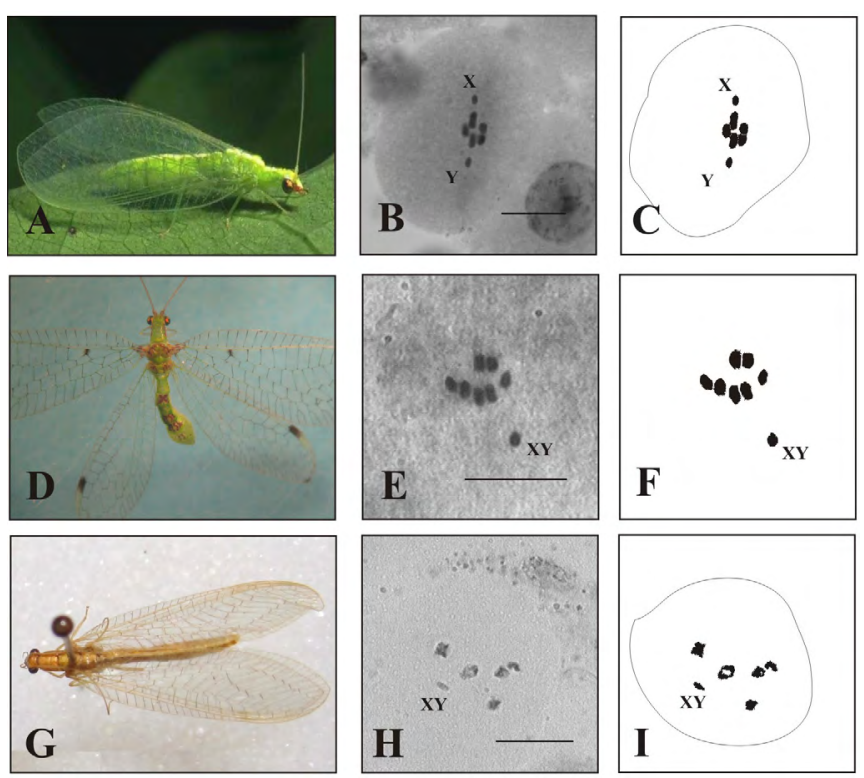

Figura 2. Neuroptera. A-C Chrysoperla externa: A. Aspecto general de un individuo en el campo. B. Metafase I, $\mathrm{n}=5+\mathrm{XY}$. C. Representación gráfica de Fig. 2B. D-F Leucochrysa cruentata: D. Aspecto general de un individuo de colección. E. Metafase I $\mathrm{n}=8+\mathrm{XY}$. F. Representación gráfica de Fig. 2E. G-I Plesiochrysa elongata: G. Aspecto general de un individuo de colección. H. Diacinesis n $=5+$ XY. I. Representación gráfica de Fig. $2 \mathrm{H}$. Escala $=10 \mu \mathrm{m}$.

El material de Euxesta eluta proviene de la cámara de cría perteneciente al laboratorio de la cátedra de Biología celular (Facultad de Ciencias Naturales e I.M.L.) y fue cedido por la Dra. M. G. Murúa.

Por cada taxón, se trabajó con 10 machos adultos fijados en la mezcla alcohol etílico - ácido acético (en proporción 3:1) y conservados posteriormente en alcohol $96 \%$. Se llevaron a cabo preparaciones microscópicas (mitóticas y meióticas) con gónadas siguiendo técnicas de citogenética clásica, mediante una gota de hematoxilina propiónica al $2 \%$ u orceína acética al $2 \%$ y tinción de fluorescencia con DAPI (4'-6-diamidino2-fenilindol) siguiendo la técnica propuesta por Schweizer (1976).

La observación y análisis tanto de las divisiones mitóticas como meióticas se efectuaron en un mínimo de siete células por cada taxón.

Las microfotografías se tomaron con una cámara Moticam 1000 (1.3MP) conectada a un microscopio Nikon Eclipse E200. Las imágenes con fluorescencia se capturaron con una cámara Olympus Color 5 conectada a un microscopio Olympus BX43. Las representaciones gráficas fueron realizadas con el software Corel Draw X3.

\section{Resultados}

En Chrysopidae (Neuroptera) los análisis revelaron dos números gaméticos diferentes: $\mathrm{n}=5+\mathrm{XY}$ en Ceraeochrysa cincta (Schneider, 1851) (Figs. 1A-1C), Ceraeochrysa paraguaria (Navás, 1920) (Figs. 1D-1F) Chrysoperla argentina (González Olazo \& Reguilón, 2002) (Figs. 1G-1I), C. asoralis (Banks, 1915) (Figs. 1J-1L), C. externa (Hagen, 1861) (Figs. 2A-2C) y Plesiochrysa elongata (Navás, 1913) (Figs. 2G-2I) donde se observaron cromosomas sexuales heteromórficos y segregación a distancia; en cambio en Leucochrysa cruentata (Schneider, 1851) las células meióticas 

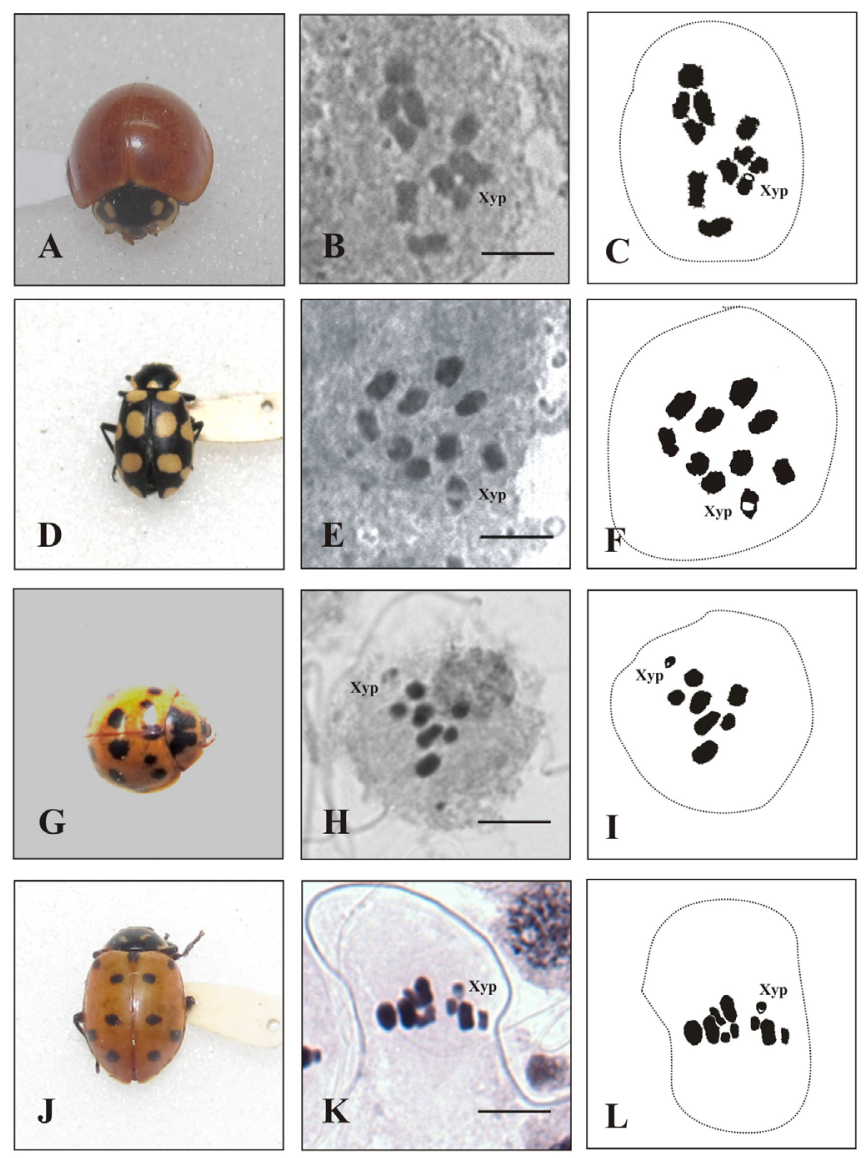

Figura 3. Coleoptera, Coccinellidae. A-C Cycloneda sanguinea: A. Aspecto general de un individuo de colección. B. diacinesis, $\mathrm{n}=9+$ $\mathrm{Xy}_{\mathrm{p}}$. C. Representación gráfica de Fig. 3B. D-F Eriopis connexa: D. Aspecto general de un individuo de colección. E. Diacinesis, $\mathrm{n}=9+$ $\left.\mathrm{Xy}_{\mathrm{p}} \mathrm{F}\right)$ representación gráfica de Fig. 3E. G-I Harmonia axyridis: G. Aspecto general de un individuo de colección. H. Metafase I $\mathrm{n}=7+$ $\mathrm{Xy}_{\mathrm{p}}$. I. Representación gráfica de Fig. 3H. J-L Hippodamia convergens: J. Aspecto general de un individuo de colección. K. Metafase I, n $=9+$ $\mathrm{Xy}_{\mathrm{p}}$, L. Representación gráfica de Fig. 3K. Escala $10 \mu \mathrm{m}$.

exhibieron $\mathrm{n}=7+\mathrm{XY}$ con cromosomas sexuales asinápticos y aquiasmáticos (Figs. 2D-2F).

En Coleoptera los datos registrados en Coccinellidae revelan que Cycloneda sanguinea (Linnaeus, 1763) (Figs. 3A-3C), Eriopis connexa (Germar, 1824) (Figs. 3D-3F), Hippodamia convergens Guerin-Meneville, 1842 (Figs. 3J$3 \mathrm{~L}$ ) presentan un número gamético de $\mathrm{n}=9+\mathrm{Xy}_{\mathrm{p}}$, mientras que en Harmonia axyridis (Pallas, 1772) se registró una fórmula meiótica de $\mathrm{n}=7+\mathrm{Xy}_{\mathrm{p}}$ (Figs. 3G-3I). En Melyridae, Astylus atromaculatus (Blanchard, 1843) exhibe una fórmula meiótica $\mathrm{n}=8+\mathrm{Xy}_{\mathrm{p}}$ (Figs. 4A-4C).

En Forficulidae (Dermaptera) el complemento cromosómico diploide de $2 \mathrm{n}=20(18 \mathrm{~A}+\mathrm{XY})$ estuvo presente en Doru lineare (Eschs, 1822) (Figs. 5A-5C) y D. luteipes (Scudder, 1876) (Figs. 5D-5F).

En Ulidiidae (Diptera) se pudo observar que en Euxesta eluta (Loew, 1868) el número gamético es $\mathrm{n}=5+\mathrm{XY}$ (Figs. 6A-6C). Los resultados se resumen en la Tabla 1.

\section{Discusión y conclusiones}

Dentro de Neuroptera los Chrysopidae son los insectos más abundantes, con alrededor de 1.300 especies reconocidas con una amplia distribución en la región Neotropical (Aspöck et al. 1980; Adams y Penny 1986; Borror et al. 1989; Brooks y Barnard 1990; Brooks 1997; Valencia Luna et al. 2006).

Los antecedentes bibliográficos mencionan para Neuroptera la presencia de segregación aquiasmática o a distancia de los cromosomas sexuales y un sistema $\mathrm{XX} / \mathrm{XY}$ con cromosomas sexuales heteromórficos (Asana y Kichijo1936; HughesSchrader 1969; Nokkala y Nokkala 2004; Andrada et al. 2012).

Séméria (1984) citó para Chrysopidae el número básico $2 \mathrm{n}$ $=12$, con variantes $2 \mathrm{n}=10$ y $2 \mathrm{n}=14$ (Hirai 1957) atribuidos a fenómenos de fusión o fisión de cromosomas.

Los resultados en las especies de Ceraeochrysa, Chrysoperla y Plesiochrysa están en total coincidencia con lo citado para la mayoría de las especies del orden. Mientras que Leucochrysa cruentata presentó un número gamético $n=7+$ $\mathrm{XY}$, en concordancia con lo publicado por Lopes (2012) para numerosas especies de Leucochrysa, incluida la especie en estudio. Los crisópidos son considerados bio-controladores de primer nivel, dada su gran capacidad depredadora y su resistencia a una amplia gama de insecticidas (Brooks y Barnard 1990; De Freitas 2002). En la región Neotropical existe un considerable interés en el grupo, particularmente en Brasil, donde se está experimentando sobre varios cultivos, como cítricos, algodón y varias hortalizas, tanto en el campo como en invernadero (De Freitas 2002). En Argentina (Tucumán) son utilizados como bio-controladores en plantaciones citrícolas (González Olazo y Reguilón 2008), recientemente incorporados en las provincias de La Rioja y Corrientes.

Hasta la actualidad, los análisis realizados en Coleoptera han demostrado que existe una gran variabilidad en el número de cromosomas, desde $2 \mathrm{n}=4$ en Chalcolepidius zonatus
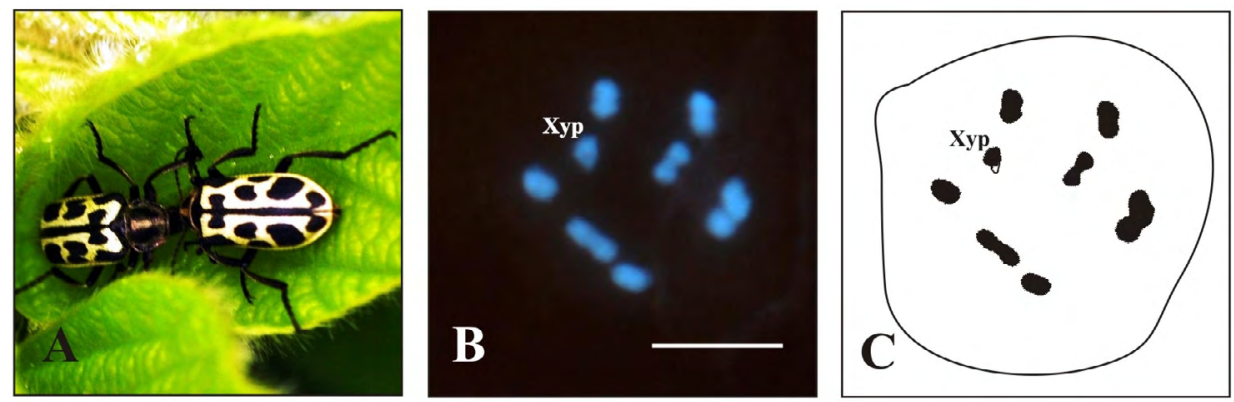

Figura. 4. Coleoptera, Melyridae. A-C Astylus atromaculatus: A. Aspecto general de individuos en el campo. B. Diacinesis, $\mathrm{n}=7+\mathrm{Xy}_{\mathrm{p}}$. C. Representación gráfica de la Fig. 4B. Escala $10 \mu \mathrm{m}$. 

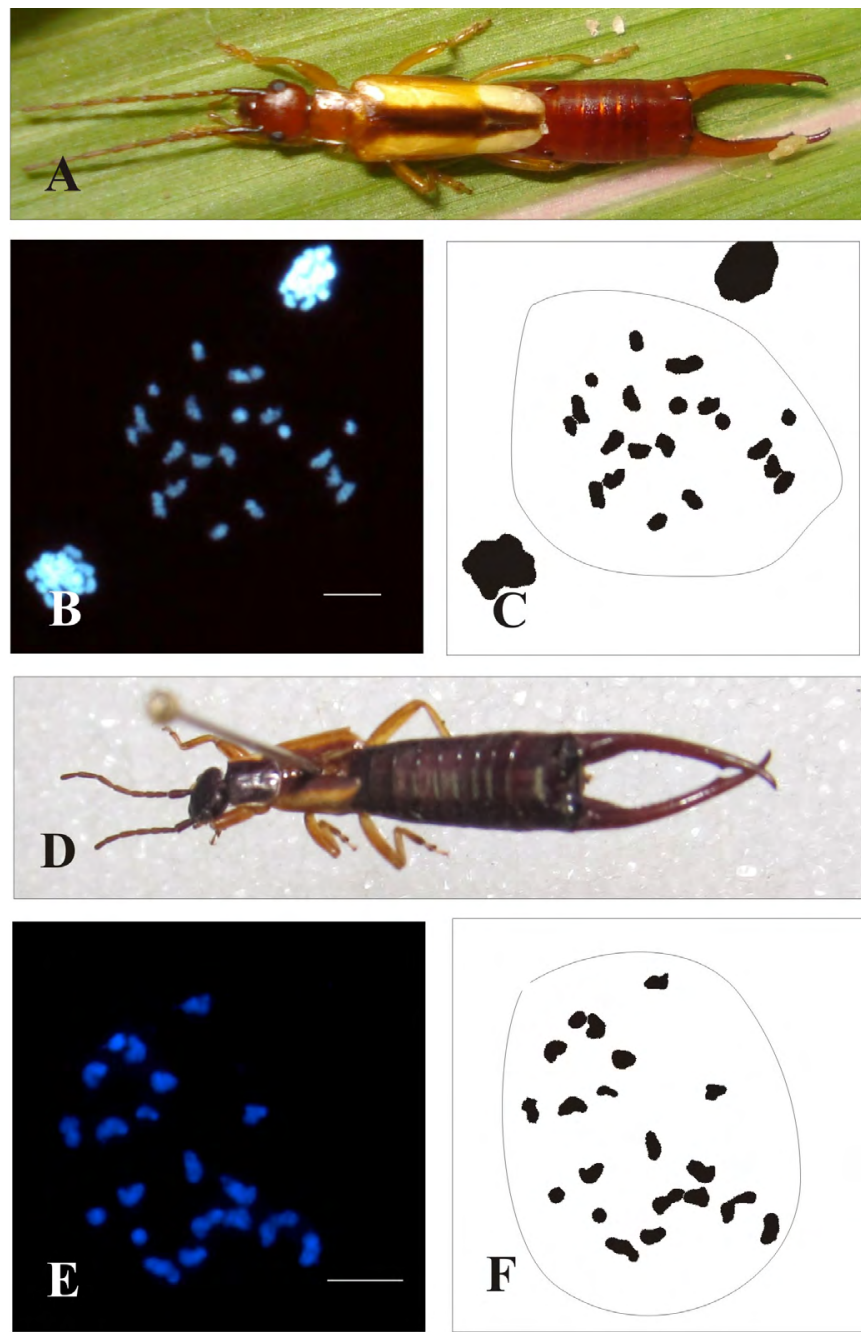

Figura 5. Dermaptera. A-C Doru lineare: A. Vista general de macho en el campo. B. Metafase mitótica $2 \mathrm{n}=20$. C. Representación gráfica de Fig. 5B; D-F. D. luteipes: D. Vista general de un individuo de colección E. Metafase mitótica, $2 \mathrm{n}=20$. F. Representación gráfica de la Fig. 5E. Escala10 $\mu \mathrm{m}$.

(Eschscholtz, 1829) (Ferreira et al. 1984), hasta $2 \mathrm{n}=69$, en Ditomus capito (Serville, 1821) (Serrano 1981).

De distribución cosmopolita, Coccinellidae, conocidas vulgarmente como "mariquitas", es una familia que cuenta con unas 6000 especies descritas y se conoce el complemento cromosómico de alrededor de 200 especies. Los números cromosómicos diploides varían entre las subfamilias entre 12 y 18, siendo el más común este último.
El sistema sexual presente en $q$ es $\mathrm{XX}$, mientras que en $\widehat{\delta}$ es $X y_{p}$, donde " $y$ " es de pequeño tamaño denominado cromosoma en paracaídas, el cual es ancestral para Coleoptera (Sloggett y Honek 2012).

Nuestros estudios en Cycloneda sanguinea, Eriopis connexa e Hippodamia convergens coinciden con citas previas para los números básicos y características cromosómicas de coleópteros (Smith 1950; Smith y Virkki 1978; Maffei et al. 2000). Para Hippodamia convergens no se encontraron antecedentes citogenéticos previos y para Harmonia axyridis se citaron recuentos cromosómicos somáticos de $2 \mathrm{n}=16$ $\mathrm{AA}+\mathrm{Xy}_{\mathrm{p}}$ en $\partial$ y $2 \mathrm{n}=16 \mathrm{AA}+\mathrm{XX}$ en hembras (Hoshiba y Niijima 1987) pero no se dan reportes meióticos, por lo que los números haploides aquí expuestos son los primeros para el género.

Las especies de coccinélidos estudiadas son consideradas de gran importancia para los agroecosistemas, ya que tanto adultos como larvas son controladores de plagas, tales como áfidos, escamas, trips, mosquitas blancas e insectos de cuerpos blandos entre otros (Morón y Terrón 1988).

La familia Melyridae Leanch (Suborden Polyphaga) cuenta con aproximadamente 5.000 especies (Leschen 2010). En la región neotropical Melyridae consta aproximadamente con 535 especies, siendo el género Astylus Laporte de Castelnau, 1836, el más representativo con 110 especies (Laporte de Castelnau 1836; Majer 1994; Constantin 2011).

En Argentina Melyridae presenta 73 especies de las cuales 62 se podrían considerar endémicas. No obstante, es altamente probable que esta información cambie cada vez que se precisen los datos de recolección y/o se revisen las colecciones de los géneros involucrados (Estrada Mancilla 2008). La representatividad de los melíridos en las colecciones es baja y sus antecedentes citogenéticos son muy escasos, no contando con registros para nuestro país.

El cariotipo haploide predominante en el suborden Polyphaga es $\mathrm{n}=9+\mathrm{Xy}_{\mathrm{p}}$, sin embargo, Astylus atromaculatus presento un número cromosómico diferente $\mathrm{n}=8+\mathrm{Xy}_{\mathrm{p}}$. Smith (1950) estudió especies de la familia Elateridae y observó alteraciones en el número de cromosomas autosómicos, ya sea por incremento (posiblemente por fragmentación) o disminución (por fusión) de éstos. Cabe la posibilidad de que la variación en el número presente en $A$. atromaculatus pueda deberse a rearreglos estructurales en la cromatina similares a los observados en Elateridae.

En cultivos de importancia económica tales como soja o girasol y en estado de larva el taxón antes mencionado se alimenta de semillas y es considerado plaga, actuando como cortador en las etapas tempranas del cultivo. En estado adulto
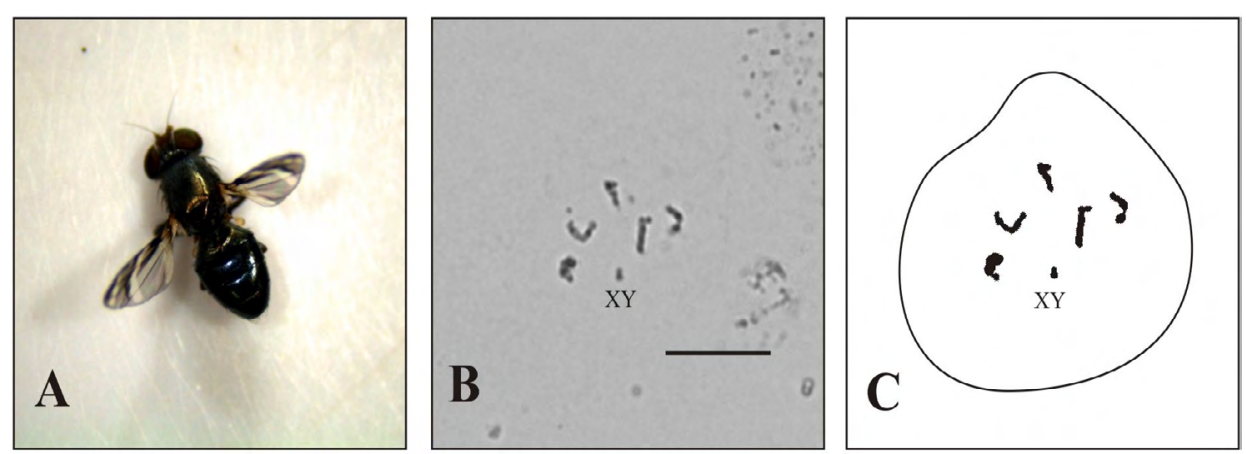

Figura 6. Diptera. Euxesta eluta: A. Aspecto general de un individuo de colección $\widehat{\partial}$. B. diacinesis, $\mathrm{n}=$ $5+$ XY. C. representación gráfica de Fig. $6 \mathrm{~B}$. Escala $=10 \mu \mathrm{m}$. 
también ocasiona daños en girasoles utilizando los capítulos para alimentarse y como sitio de apareamiento (Vitti et al. 2008).

En Dermaptera, la familia Forficulidae es de distribución cosmopolita e incluye las llamadas "tijeretas". Doru lineare y D. luteipes son especies que se distribuyen en América, desde el sur de los Estados Unidos hasta Argentina (Hincks 1949; Reichardt 1971; Mariani et al. 1996; Mariani 1998; Romero Sueldo y Virla 2009; Romero Sueldo 2012).

Los antecedentes citogenéticos indican que los cromosomas de Dermaptera carecen de constricción primaria o centrómero, por lo tanto, son holocinéticos, y el mecanismo de determinación del sexo es del tipo XX: XY (ㅇ / đ̋). Sin embargo, se observaron especies con sistemas múltiples de determinación del sexo $(2,3$ ó 4 cromosomas sexuales $\mathrm{X})$ y en ocasiones, ausencia de cromosoma «Y», así los machos se identifican sexualmente como $\mathrm{X}_{1} \mathrm{X}_{2} \mathrm{Y} ; \mathrm{X}_{1} \mathrm{X}_{2} \mathrm{X}_{3} \mathrm{Y} ; \mathrm{X}_{1} \mathrm{X}_{2}$ $\mathrm{X}_{3} \mathrm{X}_{4} \mathrm{Y}$ y $\mathrm{X}_{0}$ (Misra 1937; White 1972). En la bibliografía concerniente al orden se han determinado números cromosómicos extremos que van desde $2 \mathrm{n}=7$ Arixenia esau Jordan, 1909 (Arixeniidae) y $2 \mathrm{n}=60$ para Hemimerus bouvieri Chopard, 1934 (Hemimeriidae) (White 1971; White 1972; Mittal y Suri 1981).

Debido a la gran variación de número cromosómico en Dermaptera y a la escasa información citogenética, aún no se ha podido determinar el número cromosómico más frecuente.

La carencia de constricción primaria que hemos observado afianza la naturaleza holocinética de Doru lineare (Figs. 5A$5 \mathrm{C})$ y $D$. luteipes y la determinación del sexo concuerda con el sistema predominante en Dermaptera XX:XY.

La mayoría de los Dermaptera presenta hábitos omnívoros, cuando están en los agroecosistemas pueden considerarse insectos dañinos, actuando como organismos fitófagos, como benéficos, depredando especies plagas (Romero Sueldo y Virla 2009). Pueden encontrarse en cultivos de maíz y se mencionan como depredadores de huevos de Spodoptera frugiperda (J. E. Smith, 1797), así como de Dalbulus maidis (De Long y Wolcott, 1923) y Tapajosa rubromarginata (Signoret, 1855) (Romero Sueldo y Virla 2009).

Entre los Diptera, la familia Ulidiidae incluye a Euxesta (Loew., 1868) (Kameneva y Korneyev 1993), género que está conformado por alrededor de 90 especies y que se encuentra restringido al continente americano, excepto por pocas especies que llegan a estar ampliamente distribuidas por introducción accidental (Steyskal y Ahlmark 1995). En Argentina, Euxesta está poco estudiado, debido a su reciente aparición en la provincia de Santa Fe, causando severos daños en los maíces dulces (Bertolaccini et al. 2010). En este estudio se plantea la necesidad de nuevos estudios biológicos y agronómicos, además de sus correspondientes análisis citogenéticos. Los estudios cromosómicos en el género son escasos, cuatro especies fueron analizadas citológicamente: E. annonae (Fabricius,1794), E. eluta y E. notata (Wiedeman, 1830) con $2 \mathrm{n}=12$ y E. calligyna (Bigot, 1857) con $2 \mathrm{n}=10$ (Frías 1978; Isaev 2012); todas ellas exhiben determinación del sexo XX: XY. El número gamético de Euxesta eluta $(\mathrm{n}=6)$ se corresponde con el número diploide citado en tres especies.

De los 15 taxones analizados, se citan por primera vez antecedentes citológicos para seis de ellos: Ceraeochrysa paraguaria, Chrysoperla asoralis, Plesiochrysa elongata, Doru luteipes, Hippodamia convergens y Astylus atromaculatus; un número cromosómico gamético adicional para Euxesta eluta y un número cromosómico diferente al citado para Astylus atromaculatus. Este estudio contribuye al conocimiento y caracterización de los insectos de Argentina y en particular del NOA, poniendo en evidencia la carencia de antecedentes citogenéticos en la clase Insecta.

\section{Agradecimientos}

Queremos expresar nuestro agradecimiento a la Fundación Miguel Lillo, donde se llevó a cabo esta investigación y por el financiamiento de los viajes de recolección. A las licenciadas Mabel Romero, Mercedes Dode ${ }^{\dagger}$, Laura Almirón y a la Dra. M. G. Murúa por su inestimable colaboración en la recolección del material y fotos utilizados en este trabajo. También extendemos nuestro agradecimiento a la Dra. Emilia Constanza Pérez por poner a nuestra disposición los ejemplares de las Colección Entomológica de la Fundación Miguel Lillo y a los Sres. Francisco Sánchez y Federico Heredia por su asistencia técnica.

\section{Literatura citada}

ADAMS, P. A.; PENNY, N. D. 1986. Faunal relations of Amazonian Chrysopidae. En: Gepp, J.; Aspöck, H.; Hölzel, H. (Eds.). Recent research in neuropterology. Proceedings of the Second International Symposium on Neuropterology, Privately press., Hamburgo 119-124.

ANDRADA, A. R.; LOZZIA, M. E.; GONZÁLEZ-OLAZO, E.; HEREDIA, F. 2012. Estudios meióticos en dos especies de crisópidos (Chrysopidae: Neuroptera). Acta Zoológica Lilloana 56 (1-2): 38-43.

ANDRADA, A. R.; SILENZI, G. M.; BIGLIARDO, G. E.; ROMERO, M.; DODE, M. 2016. Estudio citogenetico en dos poblaciones naturales de Doru lineare (Dermaptera, Forficulidae). Acta Zoológica Lilloana 60 (1): 3-9.

ASANA, J. J.; KICHIJO, H. 1936. The chromosomes of six species of antlions (Neuroptera). Journal of the Faculty of Science, Hokkaido Imperial University, Series VI 5 (2): 121-136.

ASPÖCK, H.; ASPÖCK, U.; HÖLZEL, H. 1980. Die Neuropteren Europas. Eine zusammenfassende Darstellung der Systematik, Ökologie und Chorologie der Neuropteroidea Megaloptera, Raphidioptera, Planipennia) Europas. 2 Vols. Goecke und Evers. Krefeld. $495+355$ p.

BERTOLACCINI, I.; BOUZO, C.; LARSEN, N.; FAVARO, J. C. 2010. Especies del género Euxesta (Diptera: Ulidiidae=Otitidae) plagas de maíces dulces $B t$ en la provincia de Santa Fe, Argentina. Revista de la Sociedad de Entomológica Argentina 69 (1-2): 123-126.

BORROR, D. J.; TRIPLEHORN, C. A.; JOHNSON, N. F. 1989. An introduction to the study of insects. Sixth Edition. Saunders College, EE. UU. 875 p.

BRINDLE, A.; QUINTERO ARIAS, D. 1992. Earwigs of Panamá. pp. 198-207. In: Quintero Arias, D. Aiello, A. (Eds.). Insects of Panama and Mesoamerica. Selected studies. Oxford University Press, Oxford. 692 p.

BROOKS, S. J. 1997. An overview of the current status of Chrysopidae (Neuroptera) systematics. Deutsche Entomologische Zeitschrift 44: 267-275. https://doi.org/10.1002/mmnd.4800440212

BROOKS, S. J.; BARNARD, P. C. 1990. The green lacewings of the world: a generic review (Neuroptera: Chrysopidae). Bulletin of the British Museum Natural History (Entomology) 59: 117-286.

CONSTANTIN, R. 2011. A contribution to the genus Astylus Laporte de Castelnau, 1836 in Ecuador, with descriptions of three new species (Coleoptera, Melyridae). Entomologica Basiliensia 33: 39-61.

CROSLAND, M. W. J.; CROZIER, R. H. 1986. Myrmecia pilosula, an ant with only one pair of chromosomes. Science 231: 12781281. https://doi.org/10.1126/science.231.4743.1278 
DE FREITAS, S. 2002. O uso de crisopídeos no controle biológico de pragas. pp. 209-224. En: Parra, J. R. P.; Botelho, P. S. M.; Corrêa- Ferreira, B. S.; Bento J. M.S. (Eds.). Controle Biológico no Brasil: Parasitóides e Predadores, Movale, São Paulo. 635 p.

ESTRADA MANCILLA, P. 2008. Melyridae. pp. 592-602. En: Claps, L. E.; Debandi, G.; Roig-Juñent S. (Dirs.). Biodiversidad de artrópodos argentinos, volumen 2, Editorial Sociedad Entomológica Argentina, Mendoza. $615 \mathrm{p}$.

FERREIRA, A.; CELLA, D.; TARDIV, J. R.; VIRKKI, N. 1984. Two pairs of chromosomes: A new low record for Coleoptera. Revista Brasileira de Genética 7: 231-39.

FRÍAS, D. L. 1978. Citogenética de Euxesta eluta y Euxesta annonae (Diptera Otiidae). Agricultura Técnica 38 (4): 144-149.

GONZALÉZ OLAZO, E.; REGUILÓN, C. 2008. Orden Neuroptera. pp. 235-248. En: Claps, L. E.; Debandi, G.; Roig-Juñent S. (Dirs.). Biodiversidad de artrópodos argentinos, volumen 2, Editorial Sociedad Entomológica Argentina, Mendoza. 615 p.

HINCKS, W. 1949. The earwigs (Dermaptera) of Argentina. Acta Zoológica Lilloana 7: 623-652.

HIRAI, H. 1957. A cytotaxonomic study of the Chrysopidae (Neuroptera). Journal of Faculty of Science, Hokkaido Imperial University, Series VI, Zoology 13: 219-223.

HOSHIBA, H.; NIIJIMA, K. 1987. Karyological studies of two species of the Japanese coccinellid beetles, Harmonia axyridis Pallas and Menochilus sexmaculatus (Fabr.). Proceedings of the Japan Academy 63 (B): 91-92. https://doi.org/10.2183/ pjab.63.91

HUGHES-SCHRADER, S. 1969. Distance segregation and compound sex chromosomes in mantispids (Neuroptera: Mantispidae). Chromosoma 27: 109-129. https://doi. org/10.1007/BF00326139

ISAEV, V. A. 2012. Karyotypes and phylogeny of Diptera. Ivanovo State University Bulletin 2: 5-14.

KAMENEVA, E. P.; KORNEYEV, V. A. 1993. Holarctic genus Pseudoseioptera Stackelberg (Diptera: Ulidiidae (= Otitidae). Part 1. Phylogenetic relationships and taxonomic position. Journal of Ukraine Entomological Society 1: 65-72.

LAPORTE DE CASTELNAU, F. 1836. Études entomologiques, ou descriptions d'insectes nouveaux et observations sur la synonymie. Revue Entomologique 4: 5-60.

LESCHEN, R. A. B.; BEUTEL, R. G.; LAWRENCE, J. F. (Eds.). 2010. Coleoptera, Vol. II. Morphology and systematic (Elateroidea, Bostrichiformia, Cucujiformia partim): Handbook of Zoology Vol. IV, Arthropoda: Insecta. De Gruyter Press., Berlin, New York. 786 p.

LOPES, A. T. 2012. Caracterização citogenética de populações de crisopídeos (Neuroptera: Chrysopidae) de ocorrência em Jaboticabal, SP. 2012. iii, 59 f. Dissertação (mestrado) Universidade Estadual Paulista, Faculdade de Ciências Agrárias e Veterinárias, São Paulo, 70 p.

MAFFEI, E.; GASPARINO, E.; POMPOLO, S. 2000. Karyotypic characterization by mitosis, meiosis and C-banding of Eriopis connexa Mulsant (Coccinellidae: Coleoptera: Polyphaga), a predator of insect pests. Hereditas 132: 79-85. https://doi. org/10.1111/j.1601-5223.2000.00079.x

MAFFEI, E.; FRAGOSO, D. B.; POMPOLO, S.; SERRAO, J. E. 2001. Morphological and cytogenetical studies on the female and male reproductive organs of Eriopis connexa Mulsant (Coleoptera, Polyphaga, Coccinellidae). Netherlands Journal of Zoology 51 (4): 483-496. https://doi. org/10.1163/156854201X00224

MAFFEI, E.; POMPOLO, S.; PETITPIERRE, E. 2004. C-banding and fluorescent in situ hybridization with rDNA sequences in chromosomes of Cycloneda sanguinea Linnaeus (Coleoptera, Coccinellidae). Genetics and Molecular Biology 27 (2): 191194. https://doi.org/10.1590/S1415-47572004000200011

MAJER, K. 1994. A review of the classification of the Melyridae and related families (Coleoptera, Cleroidea). Entomologica Basiliensia 17: 319-390.
MARIANI, R. 1996. Catálogo de insectos fitófagos argentinos (Dermaptera). XX International Congress of Entomology, Proceedings Taxonomy of the Dermaptera, Florencia, $386 \mathrm{p}$.

MARIANI, R. 1998. Dermaptera. pp. 35-47. En: Morrone J. J.; Coscarón S. (Eds.). Biodiversidad de artrópodos argentinos, una perspectiva biotaxonómica, Ediciones Sur, La Plata. 559 p.

MARIANI, R.; VERA, L.; VIRLA, E. G. 1996. Aportes al conocimiento de Doru lineare (Eschs., 1822) (Dermaptera, Forficulidae), un insecto de importancia agronómica en el Noroeste Argentino. CIRPON. Revista de Investigación 10: 13-18.

MARIANO, C. S. F.; SANTOS, I. S.; GROC, S.; LEROY, C.; MALE, P. J.; RUIZ-GONZÁLEZ, M. X.; CERDAN, P.; DEJEAN, A.; DELABIE, J. H. C. 2011. The karyotypes of Gigantiops destructor (Fabricius) and other ants from French Guiana (Formicidae). Annales de la Société Entomologique de France (N. S.) 47: 140-146. https://doi.org/10.1080/00379271. 2011.10697705

MISRA, A. B. 1937. The chromosomes of an earwing, Forficula scudderi. The Japanese Journal of Genetics 13: 3-4. https://doi. org/10.1266/jjg.13.171

MITTAL, O. P.; SURI, V. 1981. Chromosome studies in two species of earwings (Dermaptera: Diplatyidae) from India. Caryologia 34: 441-446. https://doi.org/10.1080/00087114.1981.10796912

MORÓN, M. A.; TERRÓN, R. A. 1988. Entomología práctica. Publicación 22. Primera Edición. Instituto de Ecología A. C. México, D.F. 504 p.

NOKKALA, S.; NOKKALA, C. 2004. Interaction of B chromosomes with A or B chromosomes in segregation in insects. Cytogenetic Genome Research 106: 394-397. https:// doi.org/10.1159/000079317

NORMARK, B. B. 2003. The evolution of alternative genetic system in insects. Annual Review of Entomology 48: 397-423. https:// doi.org/10.1146/annurev.ento.48.091801.112703

PACHECO, C. A.; CHABOLI-ALEVI, K. C.; RAVAZI, A.; VILELA DE AZEREDO OLIVEIRA, M. T. 2014. Review: Malpighian tubule, an essential organ for insects. Entomology, Ornithology \& Herpetology 3 (2): 122. https://doi.org/10.4172/2161-0983.1000122

REICHARDT, H. 1971. Catologue of new world Dermaptera Forficuloidea. Papeis Avulsos do Zoologia 24: 161-184.

ROMERO SUELDO, G. M. 2012. Evaluación de Doru lineare (Dermaptera, Forficulidae) como depredador de Spodoptera frugiperda (Lepidoptera, Noctuidae). Editorial Académica Española, Saarbrücken, 1-75.

ROMERO SUELDO, M.; VIRLA, E. G. 2005. Forage preference of Doru lineare (Eschs.) (Dermaptera: Forficulidae) under laboratory conditions. Revista de la Sociedad de Entomología Argentina 64: 430-431.

ROMERO SUELDO, M.; VIRLA, E. G. 2009. Doru lineare (Dermaptera: Forficulidae), insecto benéfico en cultivos de maíz del norte argentino: preferencias alimenticias y tasas de consumo. Boletín de Sanidad Vegetal - Plagas 35: 39-47.

ROMERO SUELDO, M.; BENÍTEZ DE PARRA, L.; TORRES DE LA PLAZA, M. I. 2005. Duración y supervivencia de los estadios ninfales de Doru lineare (Dermaptera: Forficulidae) sometidos a diferentes dietas. Acta Zoológica Lilloana 49: 59-63.

ROMERO SUELDO, M.; BRUZZONE, O. A.; VIRLA, E. G. 2010. Characterization of Doru lineare (Eschscholtz) (Dermaptera - Forficulidae) as predator of fall armyworm larvae (Lep. - Noctuidae): a functional response study. Journal of Insect Science 10: 1536-2442. https://doi.org/10.1673/031.010.3801

SÉMÉRIA, Y. 1984. Some karyotypes in Chrysopidae. pp. 4248. En: Spencer K. A. (Ed.). Biology of Chrysopidae. Series Entomologica, 27, Dr. W. Junk Press., Londres, 294 p.

SERRANO, J. 1981. Chromosome numbers and karyotype evolution of Caraboidea. Genetica 55: 51-60. https://doi.org/10.1007/ BF00134005

SCHWEIZER, D. 1976. Reverse fluorescent chromosome banding with chromomycin and DAPI. Chromosoma 58: 307-324. https://doi.org/10.1007/BF00292840 
SLOGGETT, J. J.; HONEK, A. 2012. Ecology and behaviour of the ladybird beetles (Coccinellidae), First Edition. Hodek I., Van Emden H.F., Honek. A. Eds. Wiley-Blackwell Press., Oxford. $565 \mathrm{p}$.

SMITH, S. 1950. The cytotaxonomy of Coleoptera. Canadian Entomologist 82 (3): 58-68. https://doi.org/10.4039/Ent8258-3

SMITH, S. G.; VIRKKI, N. 1978. Animal cytogenetics III. Insecta V. Coleoptera. Gebrüder Borntraeger, Berlin-Stuttgart, 366 p.

STEYSKAL, G. C.; AHLMARK, K. M. 1995. Two new species of Euxesta Loew (Diptera: Otitidae). Insecta Mundi 9: 189-192.

VALENCIA LUNA, L. A.; ROMERO NÁPOLES, J.; VALDEZ CARRASCO, J.; CARRILLO SÁNCHEZ，J. L.; LÓPEZ MARTÍNEZ, V. 2006. Taxonomía y registro de Chrysopidae (Insecta: Neuroptera) en el estado de Morelos, México. Acta Zoológica Mexicana 22 (1): 17-61.

VIRGILIO, M.; BACKELJAU, T.; NEVADO, B.; DE MEYER, M. 2010. Comparative performances of DNA barcoding across insect orders. BMC Bioinformatics 11: 206. https://doi. org/10.1186/1471-2105-11-206

VITTI, D.; SALTO, C.; SOSA, M. A.; LUISELLI, S. 2008. Insectos en girasol: polinizadores, fitófagos y entomófagos. Ediciones INTA, Argentina, $54 \mathrm{p}$.

WHITE, M. J. 1971. The chromosomes of Hemimerus bouvieri Chopard (Dermaptera). Chromosoma 34 (2): 183-189. https:// doi.org/10.1007/BF00285185
WHITE, M. J. 1972. The chromosomes of Arixenia essau Jordan (Dermaptera). Chromosoma 36 (4): 338-342. https://doi. org/10.1007/BF00336792

\section{Origen y financiación}

Este estudio fue apoyado por la Fundación Miguel Lillo en el marco del proyecto B0013-1 (Miguel Lillo 251, T4000JFE, Tucumán).

\section{Contribución de los autores}

Todos los autores formaron parte del diseño de la presente investigación y la colección del material. Valeria de los A. Páez y Aldo $R$. Andrada realizaron las preparaciones citogenéticas en Chrysopidae, Gabriela M. Silenzi Usandivaras y Aldo R. Andrada, en Dermaptera, María M. Moreno Ruiz Holgado y Graciela Ruiz de Bigliardo en Coleoptera y Valeria de los A. Páez y Andrea Oviedo en Diptera, aplicando técnicas citológicas convencionales. Los autores tuvieron igual participación en la adquisición de datos y su interpretación. Valeria de los A. Páez, Aldo R. Andrada, Gabriela M. Silenzi Usandivaras, María M. Moreno Ruiz Holgado, Andrea Oviedo y Graciela Ruiz de Bigliardo contribuyeron a la discusión y revisión del manuscrito final. 